\title{
NUEVAS ESPECIES DE HISTRIGNÁTIDOS (THELASTOMATOIDEA: HYSTRIGNATHIDAE) DE LA SIERRA DEL ROSARIO, PINAR DEL RÍO, CUBA
}

\author{
Nayla García Rodríguez ${ }^{1}$, Luisa Ventosa Zenea ${ }^{1}$ y Jans Morffe Rodríguez ${ }^{2}$ \\ 1. Instituto de Ecología y Sistemática. Carretera de Varona, km 31/2, Capdevila, Boyeros, Ciudad de La Habana, \\ Cuba. \\ nayla@ecologia.cu, mluisa@ecologia.cu \\ 2. Instituto de Investigaciones en Fruticultura Tropical. Ave. 7 ma. \#3205 e/ 30 y 32, Playa, Ciudad de La Habana, \\ Cuba. \\ jmorffe@gmail.com
}

\section{RESUMEN}

Se describen dos nuevas especies de histrignátidos (Thelastomatoidea: Hystrignathidae), parásitas de Passalus interstitialis y P. pertyi (Insecta: Coleoptera), respectivamente, para la Sierra del Rosario, Pinar del Río, Cuba. Otras dos especies, Longior zayasi Coy, García et Álvarez, 1993 y Salesia cubana García et Coy, 1995, se registran por primera vez para esta localidad.

Palabras clave: especies nuevas, histrignátidos, Hystrignathus, Passalidae, Cuba.

\section{ABSTRACT}

Two new species of hystrignathids (Thelastomatoidea: Hystrignathidae); parasites of Passalus interstitialis and P. pertyi (Insecta: Coleoptera), respectively, are described from Sierra del Rosario, Pinar del Río, Cuba. Two other species, Longior zayasi Coy, García et Álvarez, 1993 and Salesia cubana García et Coy, 1995, are reported for the first time for this locality.

Key words: new species, hystrignathids, Hystrignathus, Passalidae, Cuba.

\section{INTRODUCCIÓN}

Las primeras referencias de nemátodos parásitos de pasálidos se deben a Leidy (1850), quien describe a Hystrignathus rigidus Leidy, 1850, como nuevo género y especie, parásito de Passalus cornutus (Coleoptera: Passalidae) del este de Norteamérica. En Cuba, sin embargo, los estudios referentes a la helmintofauna asociada a pasálidos son muy recientes. Los primeros histrignátidos registrados para la isla se corresponden con Hystrignathus inflatus Travassos et Koss, 1957 y Longior longicollis Travassos et Kloss, 1958, descritos de Brasil y una nueva especie, Hystrignathus pinarensis Coy, 1990, parásitas de Passalus interstitialis Scholtz, 1829 (Coy, 1990). Coy et al. (1993) describen dos nuevos taxones: Glaber poeyi Coy, García et Álvarez, 1993 y Longior zayasi Coy, García et Álvarez, 1993, este último a partir de la revisión del material asignado con anterioridad a L. longicollis. García y Coy $(1994 ; 1995 \mathrm{a}, \mathrm{b})$ describen otras tres especies: Longior alius García et Coy, 1994, Salesia cubana García et Coy, 1995 y Artigasia simplicitas García et Coy, 1995, las dos primeras parásitas de Passalus pertyi Kaup, 1869. Trabajos recientes registran la presencia de otras tres nuevas especies de histrignátidos en $P$. pertyi de la Isla de la Juventud (García, Ventosa y Morffe, en prensa).

En este trabajo se describen dos nuevas especies de histrignátidos para la Sierra del Rosario y se registra la presencia de Longior zayasi y Salesia cubana.

\section{MATERIALES Y MÉTODOS}

Para este estudio se colectó de forma manual, en troncos en descomposición, un ejemplar de Passalus pertyi Kaup, 1869 (loma El Mulo, Sierra del Rosario, Pinar del Río, Cuba; octubre, 2007). Se sacrificó en etanol al 70\% y se conservó en el mismo para su traslado al laboratorio.

El intestino se extrajo mediante cortes en las membranas pleurales del abdomen y se colocó en una placa Petri con agua, para prevenir la deshidratación del material. Luego se procedió a su disección bajo el microscopio estereoscópico con ayuda de pinzas de punta fina y agujas enmangadas. El contenido se lavó varias veces hasta separar los parásitos. Se tomó nota de la posición de los nemátodos en el tracto intestinal. La conservación definitiva se llevó a cabo en viales con etanol al 70\%. Además, se examinó material depositado en colecciones provenientes de Passalus interstitialis Escholtz, 1829 de loma El Salón, Sierra del Rosario, Pinar del Río, Cuba. 
Para su examen, los nemátodos se montaron de forma temporal en portaobjetos con glicerina anhidra. La observación se llevó a cabo en un microscopio clínico Olympus, a un aumento entre 40x y 1000x. Las variables morfométricas medidas fueron las propuestas por Travassos y Kloss (1958). Para ello se empleó un micrómetro ocular de escala lineal con una precisión de $0,001 \mathrm{~mm}$, acoplado al microscopio. Para la identificación de los nemátodos se siguió el sistema propuesto por Adamson y Van Waerebeke (1992) y De Ley y Blaxter (2002). El sistema reproductor se clasificó según la terminología de Chitwood y Chitwood (1974).

Los dibujos de cada especie se realizaron utilizando una cámara clara acoplada a un microscopio clínico Carl Zeiss. Con posterioridad, se digitalizaron y retocaron mediante los programas CorelDRAW 12 y Adobe Photoshop 7.0. En ambas especies se esquematizó la región cefálica hasta el comienzo del intestino, la región de la vulva con los huevos y la región caudal a partir del ano. Las escalas de las ilustraciones están dadas en milímetros.

El material tipo se encuentra depositado en la Colección Helmintológica de las Colecciones Zoológicas del Instituto de Ecología y Sistemática, La Habana, Cuba (CZACC).

\section{RESULTADOS}

\section{SISTEMÁTICA}

Hystrignathidae

Hystrignathus Leidy, 1850.

Hystrignathus coyi sp. nov.

(Fig.1)

Hystrignathus inflatus Travassos et Kloss, 1957, Coy, 1990:3-5, fig. 2, error de identificación.

Diagnosis. Dilatación cefálica característica a continuación del labio. Espinas desde el final de la dilatación cefálica hasta cerca del final del cuerpo esofágico. Alas laterales desde el nivel del poro excretor postbulbar hasta el nivel de la vulva. Longitud de la cola mayor que la longitud del esófago.

Diagnosis. A typical swollen cephalic region behind the lip. Spines starting from the posterior part of the cephalic swollen area to the vicinity of the procorpus. Lateral alae extending from the vicinity of the excretory pore (situated behind the bulb) to the level of the vulva. Tail longer than esophagus.

Descripción. Nemátodos de pequeño tamaño, con el cuerpo fusiforme. Cutícula con estrías transversales marcadas, hasta cerca de la base de la cola. Boca con labio anular provisto de ocho papilas pareadas. A continuación presenta una dilatación cuticular lisa, en forma de cono truncado, que abarca aproximadamente la mitad de la longitud del estoma. Región cervical con espinas dispuestas en hileras no alternadas, a partir de la dilatación cefálica hasta casi el final del cuerpo esofágico. Estoma cilíndrico y alargado. Cuerpo esofágico claviforme, con su porción posterior poco ensanchada. Anillo nervioso no observado. Istmo bien diferenciado. Bulbo esférico y musculoso, con aparato valvular bien desarrollado. Intestino subrectilíneo, algo ensanchado en su porción anterior, ano poco sobresaliente. Alas laterales desarrolladas, desde el nivel del poro excretor hasta el nivel de la vulva. Sistema reproductor didelfo anfidelfo. Vulva hacia la mitad del cuerpo, transversal y poco sobresaliente. Pocos huevos por hembra, máximo 4, elipsoidales y de cáscara lisa. Cola cónica, algo subulada, con su extremo puntiagudo. Machos desconocidos.

Tipos. Holotipo (hembra) CZACC 11.4441, CUBA, Pinar del Río, Sierra del Rosario, loma El Salón, II.1989, col. A. Coy y M. Álvarez, en Passalus interstitialis. Paratipos (4 hembras) CZACC 11.44424445, idénticos datos que el holotipo.

Medidas del Holotipo (paratipos entre paréntesis). Longitud total 2.925 (2.175-2.775). Ancho máximo $0.150(0.125-0.150)$. Longitud del estoma 0.068 (0.055-0.063). Longitud del cuerpo esofágico 0.330 (0.290-0.320). Longitud del istmo 0.053 (0.038-0.048). Diámetro del bulbo $0.073(0.068-0.078)$. Distancia ano-cola 0.725 (0.600-0.690). Distancia vulva-cola 1.530 (1.200-1.330). Huevos, 8 (3 de ellos en el holotipo), $0.113-0.125 \times 0.035-0.048(0.095-0.113 \times 0.033-0.043)$.

Hospedante. Passalus interstitialis (Insecta: Coleoptera). Localización, ciegos del intestino posterior. 

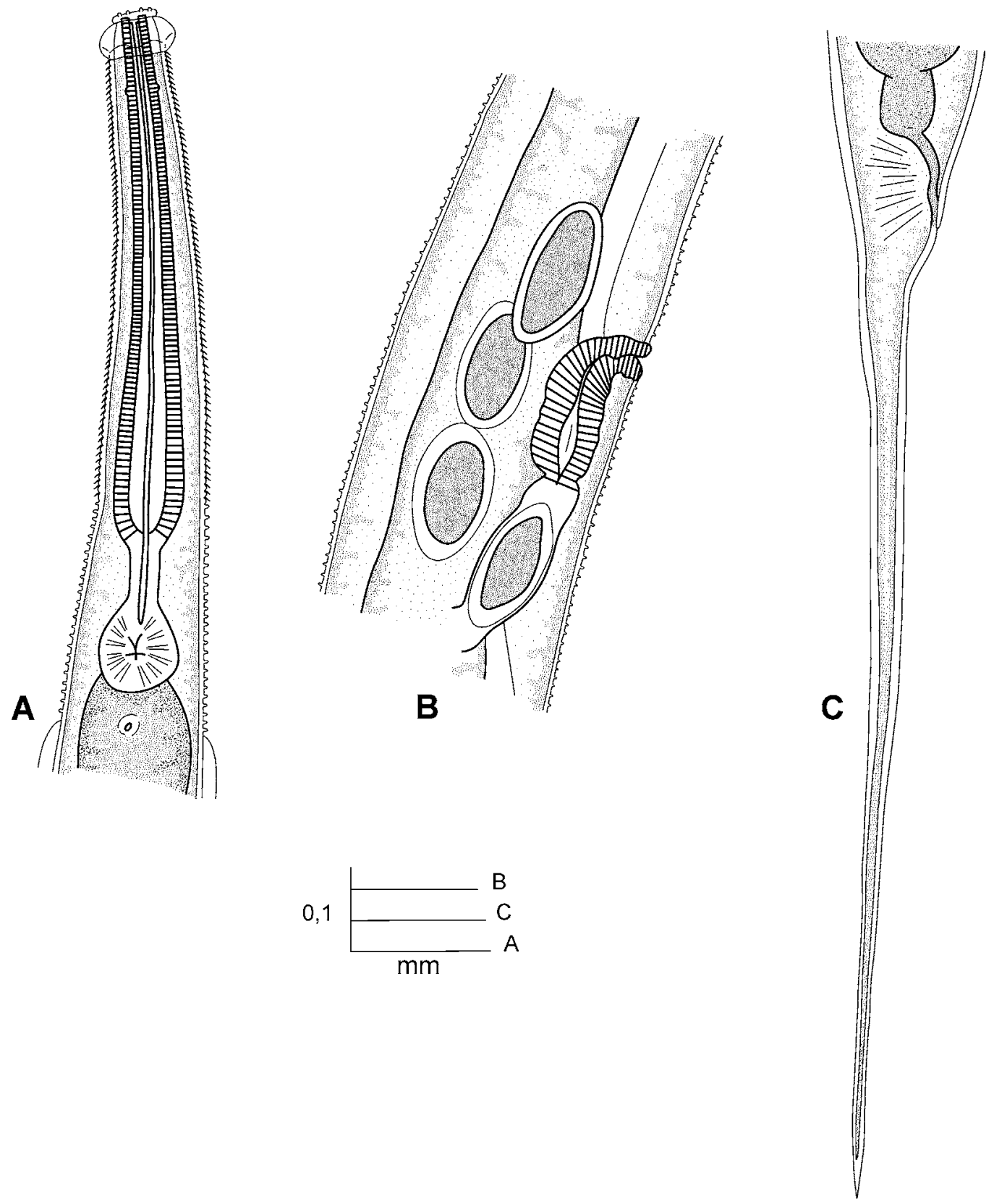

Figura 1. Hystrignathus coyi sp. nov. (hembra). A-Región cefálica. B-Vulva, huevos. C-Región caudal.

Discusión. Hystrignathus coyi sp. nov., se diferencia de la mayoría de las especies del género por la dilatación cefálica, carácter que comparte sólo con H. inflatus Travassos et Kloss, 1957 y H. tarda Artigas, 1926, ambas de Brasil (Artigas, 1926; Travassos y Kloss, 1957a). De la primera, con cola y esófago de igual longitud y alas laterales desde el fin de las espinas, difiere en poseer la cola con mayor longitud que el esófago y alas laterales que parten del nivel del poro excretor, a cierta distancia del final de las espinas. En la segunda, la cola es más corta y el esófago mucho más elongado. Especímenes procedentes de la localidad tipo fueron identificados por Coy (1990) como H. inflatus, debido a sus marcadas semejanzas con el taxon brasileño.

Etimología. Patronímico en honor al Dr. Alberto Coy Otero, destacado parasitólogo cubano e iniciador de los estudios referentes a la helmintofauna asociada a pasálidos de Cuba. 


\section{Hystrignathus rosario sp. nov.}

(Fig.2)

Diagnosis. Espinas cervicales desde el final del segundo anillo cefálico hasta el nivel del poro excretor postbulbar. Alas laterales desde el final de las hileras de espinas hasta el nivel de la vulva. Huevos elipsoidales ornamentados por crestas longitudinales.

Diagnosis. Cervical spines starting behind the second cephalic ring to the vicinity of the excretory pore. Lateral alae extended from the end of the spine rows to the vicinity of the vulva. Elipsoidal eggs ornamented with longitudinal ridges.

Descripción. Nemátodos de mediano tamaño, robustos y fusiformes. Cutícula con estrías transversales, más marcadas hacia la región anterior. Labio anular provisto de ocho papilas dispuestas en cuatro pares. Primero y segundo anillos cefálicos de ancho similar. Región cervical con espinas dispuestas en hileras no alternadas hasta el nivel del poro excretor postbulbar. Estoma cilíndrico. Cuerpo esofágico claviforme, con su porción posterior poco dilatada. Anillo nervioso en la mitad del cuerpo esofágico. Istmo bien diferenciado. Bulbo esférico con aparato valvular desarrollado. Intestino subrectilíneo, ensanchado en su porción anterior. Alas laterales bien desarrolladas, desde el final de las hileras de espinas hasta el nivel de la vulva. Sistema reproductor didelfo anfidelfo. Vulva poco sobresaliente. Huevos, 4 a 7 por hembra, elipsoidales y ornamentados con crestas longitudinales. Cola cónica y alargada, sin transición nítida. Machos desconocidos.

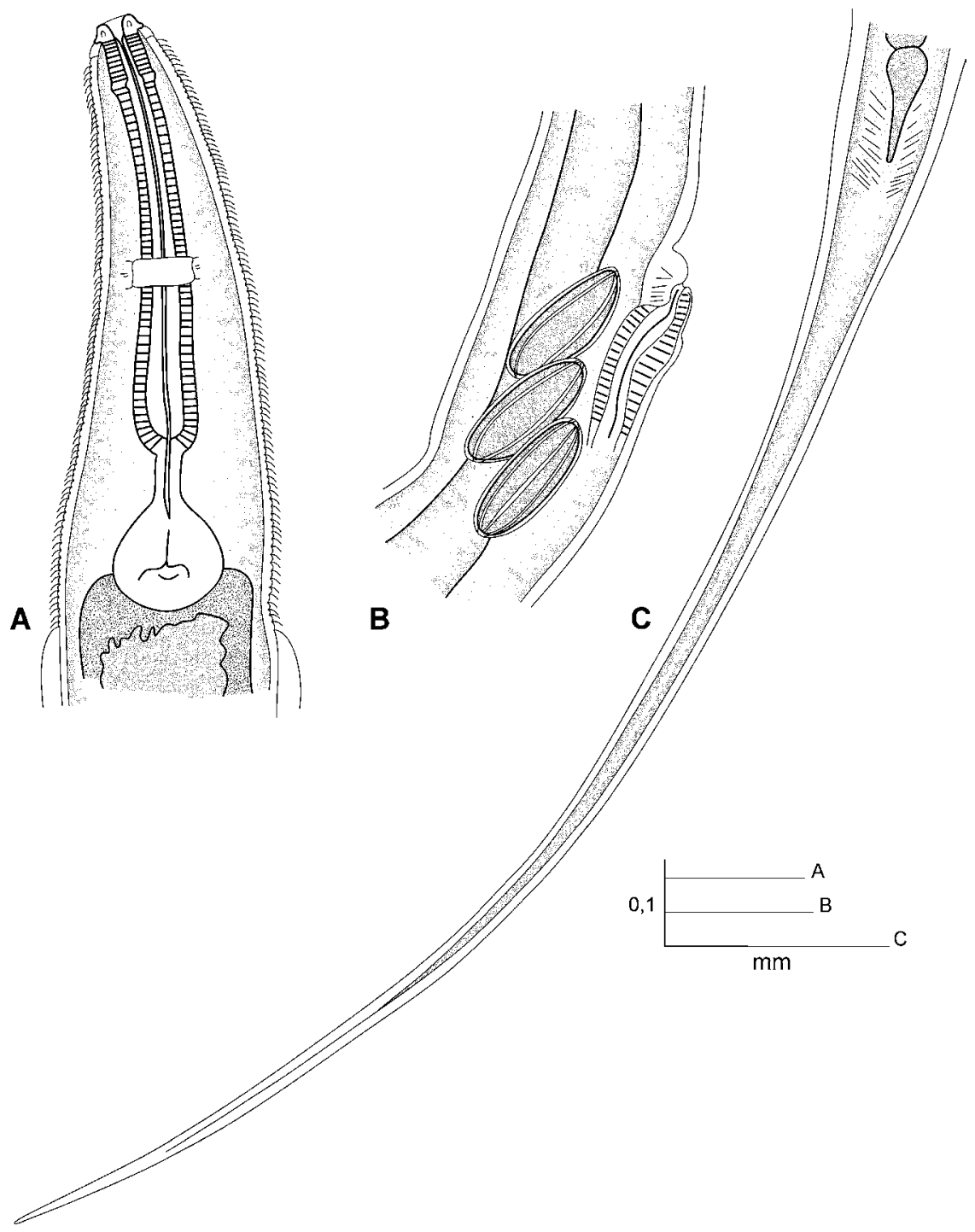

Figura 2. Hystrignathus rosario sp. nov. (hembra). A-Región cefálica. B-Vulva, huevos. C-Región caudal. 
Tipos. Holotipo (hembra) CZACC 11.4425, CUBA, Pinar del Río, Sierra del Rosario, loma El Mulo, X.2007, col. R. Núñez y O. Madruga, en Passalus pertyi. Paratipos (9 hembras) CZACC 11.4426-4435, idénticos datos que el holotipo.

Medidas del Holotipo (paratipos entre paréntesis). Longitud total 2.225 (1.950-2.325). Ancho máximo $0.130(0.120-0.180)$. Longitud del estoma $0.040(0.030-0.043)$. Longitud del cuerpo esofágico 0.250 (0.220-0.270). Distancia del anillo nervioso al extremo anterior $0.180(0.173-0.190)$. Longitud del istmo 0.045 (0.028-0.048). Diámetro del bulbo 0.068 (0.065-0.088). Distancia ano-cola $0.600(0.510-0.650)$. Distancia vulva-cola 1.175 (0.900-1.200). Huevos, 26 ( 3 de ellos en el holotipo), $0.100-0.103 \times 0.038-0.043$ $(0.100-0.123 \times 0.025-0.043)$.

Hospedante. Passalus pertyi (Insecta: Coleoptera). Localización: ciegos del intestino posterior.

Discusión. Hystrignathus rosario sp. nov. se diferencia de H. inflatus Travassos et Kloss, 1957 y H. tarda Artigas, 1928, por carecer de la dilatación cefálica característica de estos (Artigas, 1928; Travassos y Kloss, 1957a). Por la extensión de las espinas difiere de $H$. cobbi Travassos et Kloss, 1957; H. egalis Van Waerebeke et Remillet, 1982; H. heliae Travassos et Kloss, 1957; H. inegalis Van Waerebeke et Remillet, 1982; H. insularis Van Waerebeke, 1973; H. meridensis Guerrero, 1980; H. pinarensis Coy, 1990; H. popiliophagus Guerrero, 1980 y H. rescens Travassos et Kloss, 1958; especies en las que las espinas no sobrepasan el nivel del bulbo (Travassos y Kloss, 1957b; 1958; Guerrero, 1980; Van Waerebeke y Remillet, 1982; Coy, 1990). La ornamentación de los huevos lo separa de H. rigidus Leidy, 1850; H. pearsoni Travassos et Kloss, 1958 e H. spinosus Travassos et Kloss, 1957, especies con huevos de cáscara lisa (Leidy, 1850; Travassos y Kloss, 1957a; 1958). De H. ferox Hunt, 1982 (Hunt, 1982), se diferencia por la extensión de las alas laterales, que terminan al nivel de la vulva en la especie cubana y finalizan a aproximadamente dos veces el ancho del cuerpo antes de la vulva; en la otra. De H. rugosus Travassos et Kloss, 1958 (Travassos y Kloss, 1958) difiere por la mayor longitud de su cola, cerca de un cuarto de la longitud total y por su esófago más corto. Hystrignathus rosario sp. nov. guarda mucha semejanza desde el punto de vista morfológico y morfométrico con $H$. spinosus, pero la ornamentación de sus huevos permite su fácil diferenciación.

Etimología. Nombre en aposición referido al macizo montañoso donde se ubica la localidad tipo de la especie.

Comentario. Otros registros en la muestra examinada fueron la presencia de Longior zayasi Coy, García et Álvarez, 1993 y Salesia cubana García et Coy, 1995.

\section{AGRADECIMIENTOS}

Al MSc. Rayner Núñez, del Instituto de Ecología y Sistemática y la Licda. Ormaily Madruga, del Museo Nacional de Historia Natural, por la colecta de los coleópteros. A la Dra. Ileana Fernández, también del Instituto de Ecología y Sistemática, por la identificación de los hospedantes. Este resultado está inscrito en el proyecto Colecciones Zoológicas, su conservación y manejo, del Programa Ramal de Ciencia y Técnica Diversidad Biológica de la Agencia de Medio Ambiente (AMA) del Ministerio de Ciencia, Tecnología y Medio Ambiente (CITMA).

\section{LITERATURA CITADA}

Adamson, M. y D. Van Waerebeke. 1992. Revision of the Thelastomatoidea, Oxyurida of invertebrate hosts III. Hystrignathidae. Syst. Parasit. 22: 111-130.

Artigas, P. 1926. Nematodes de invertebrados I. Bol. Biol., Sao Paulo 1: 1-13.

Artigas, P. 1928. Nematodes de invertebrados. Bol. Biol., Sao Paulo 3: 71-75.

Chitwood, B. G. y M. G. Chitwood. 1974. Introduction to Nematology. University Park Press, Baltimore, $334 \mathrm{pp}$. 
Coy, A. 1990. Nemátodos de la familia Hystrignathidae parásitos de coleópteros (Passalidae) de Cuba. Poeyana 402: 1-7.

Coy, A., N. García y M. Álvarez. 1993. Nemátodos parásitos de insectos cubanos, Orthoptera (Blattidae y Blaberidae) y Coleoptera (Passalidae y Scarabaeidae). Acta Biol. Venez. 14(3): 53-67.

De Ley, P. y M. Blaxter. 2002. Systematic position and phylogeny. En: Biology of Nematodes (Ed.: D. L. Lee) Taylor \& Francis, Londres, 1-30.

García, N. y A. Coy. 1994. Descripción de dos nuevas especies y registro de nuevos hospederos de nemátodos (Nematoda) de la región oriental de Cuba. Avicennia 1:13-17.

García, N. y A. Coy.1995a. Nemátodos parásitos de artrópodos de la Sierra de los Órganos, Cuba. AvaCient $14: 26-30$.

García, N. y A. Coy.1995b. Nuevas especies de nemátodos (Nematoda) parásitos de artrópodos cubanos. Avicennia 3: 87-96.

García, N., L. Ventosa y J. Morffe. En prensa. Primeros registros de histrignátidos (Oxyurida: Thelastomatoidea: Hystrignathidae) para la Isla de la Juventud, Cuba. Solenodon.

Guerrero, R. 1980. Descripción de cuatro especies nuevas de Thelastomatidae (Nematoda) endoparásitos de Popilius sp. (Coleoptera: Passalidae) de Los Andes venezolanos. Mems. Soc. Ciens. Natur. (La Salle) 40(114): 63-78.

Hunt, D. J. 1982 Hystrignathus ferox n. sp. and Xyo xiphacanthus n. sp. (Oxyurida: Hystrignathidae) with additional data on Carlosia tijucana Travassos and Kloss, 1957. Syst. Parasit. 4: 59-68.

Leidy, J. 1850. Description of some nematoid Entozoa infesting insects. Proc. of the Acad. of Nat. Sci., Philadelphia 5: 100-102.

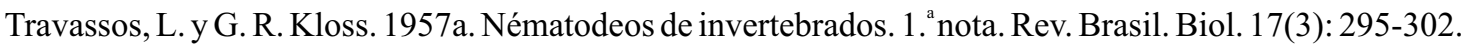

Travassos, L. y G. R. Kloss. 1957b. Nématodeos de invertebrados. 2. e 3. ${ }^{a}$ notas. Rev. Brasil. Biol. 17(4): 467-477.

Travassos, L. y G. R. Kloss. 1958. Sobre a fauna de Nematodeos dos Coleopteros-Passalidae da Estacao biologica de Boraceia. Arq. de Zool., Sao Paulo 11: 23-57.

Van Waerebeke, D y M. Remillet. 1982. Redescription de deux espéces d'Hystrignathus et redefinition du genre (Nematoda: Oxyuroidea). Revue de Nematologie 5: 285-294. 